\title{
Criminologie
}

\section{Adolescents et délinquance sexuelle}

\section{Suzette Laforest et Richard Paradis}

Volume 23, numéro 1, 1990

Nouvelles violences à l'adolescence

URI : https://id.erudit.org/iderudit/017289ar

DOI : https://doi.org/10.7202/017289ar

Aller au sommaire du numéro

Éditeur(s)

Les Presses de l'Université de Montréal

ISSN

0316-0041 (imprimé)

1492-1367 (numérique)

Découvrir la revue

Citer cet article

Laforest, S. \& Paradis, R. (1990). Adolescents et délinquance sexuelle. Criminologie, 23(1), 95-116. https://doi.org/10.7202/017289ar

\section{Résumé de l'article}

Our work with young offenders at the Quebec Social Service Centre has led us to intervene more and more frequently among adolescents for sex-related offences. We felt the need, therefore, to know more about the matter in order to be able to answer the needs of this clientèle more realistically. Thus we conceived of group therapy centred on the psychosexual development of these adolescents, along with complementary individual treatment.

A first experiment was undertaken in the spring of 1989. The results encourage us to pursue this type of treatment, always adjusting it to the needs underlying the behaviour of these adolescents.

It must be pointed out that the data and the thinking contained in this article are drawn from our practice. It is as social workers that we deal with this problem. 
Our work with young offenders at the Quebec Social Service Centre has led us to intervene more and more frequently among adolescents for sex-related offences. We felt the need, therefore, to know more about the matter in order to be able to answer the needs of this clientele more realistically. Thus we conceived of group therapy centred on the psychosexual development of these adolescents, along with complementary individual treatment.

A first experiment was undertaken in the spring of 1989. The results encourage us to pursue this type of treatment, always adjusting it to the needs underlying the behaviour of these adolescents.

It must be pointed out that the data and the thinking contained in this article are drawn from our practice. It is as social workers that we deal with this problem.

\begin{abstract}
«L'homme ne peut se définir que dans une relation symbolique avec un univers où il lui faut à la fois développer une cohérence interne et respecter la cohérence de son voisin. Il ne peut trouver une satisfaction réelle dans une expression anarchique de son érotisme, comme il ne peut pas trouver le bonheur dans un monde où il multipliera les expériences contradictoires sans savoir pourquoi il le fait. Si nous pouvons effectivement annihiler l'enfant par un schérna rigide dans lequel il doit s'inscrire sous peine d'être condamné, nous pouvons tout aussi sûrement l'aliéner en refusant de le guider dans sa quête d'une identité, qu'elle soit génitale ou autre.»
\end{abstract}

LEMAY, Michel. Psychopathologie juvénile. Tome II, Éd. Fleuries, Paris, 1973, pp. 895-989.

\title{
INTRODUCTION
}

Le concept de délinquance juvénile recouvre le très large éventail des comportements antisociaux liés à diverses problématiques, dont celles de la toxicomanie, de la violence ou encore des désordres sexuels. Depuis quelques années, la délinquance sexuelle à l'adolescence est devenue plus visible, plus préoccupante.

1. Intervenants, Service aux jeunes contrevenants du Centre de services sociaux de Québec, 540 boul. Charest est, C.P. 3910 Succursale St-Roch, Québec, Qc G1K 8L6 
Denis Lafortune ${ }^{2}$ rapporte que l'abus sexuel déclaré serait le crime dont le taux augmente le plus rapidement aux États-Unis et au Québec. Ainsi Agedon (1983), malgré les difficultés d'établir des statistiques précises, est parvenu à estimer qu'aux États-Unis seulement, de 195000 à 450000 adolescents commettent des agressions sexuelles chaque année. De plus, l'étude des populations plus âgées démontre qu'environ la moitié des délinquants sexuels identifiés à l'âge adulte ont posé leurs premiers gestes avant l'âge de 18 ans (Abel et al., 1985; Groth, 1979).

Deux inventaires de clientèle d'adolescents auteurs de délits sexuels effectués au Centre de services sociaux de Québec entre 1986 et 1988 nous ont permis de constater une nette augmentation de ce type de références. En effet, le nombre de ces situations est passé de 22 en $1986^{3}$ à 40 en $1988^{4}$, soit une augmentation de près de $100 \%$. Diverses hypothèses peuvent expliquer un tel accroissement des adolescents référés à nos services pour des agressions sexuelles. Nous croyons que les amendements au Code criminel, les orientations de services préconisant une dénonciation policière, une plus grande sensibilisation de la population à la connaissance du phénomène des abus sexuels et aux impacts de ce type de délinquance ont contribué à l'augmentation constatée.

Cette plus grande visibilité du phénomène de même que notre pratique quotidienne ont suscité un intérêt grandissant face au traitement de ces jeunes qu'on appelle souvent «abuseurs sexuels». Nous avons cherché à mieux comprendre le problème afin de trouver une réponse au défi que nous pose, en tant qu'intervenants, cette question de la délinquance sexuelle chez les adolescents.

Cette réponse, le type d'intervention développé, est en fait le fruit du travail et de la réflexion de nombreux intervenants du Centre de services sociaux de Québec. Ceux-ci, dans le cadre de leur pratique d'évaluation et de prise en charge des adolescents référés et dans le cadre également de groupes de travail, ont en effet souligné la nécessité d'une intervention spécifique concernant le développement psychosexuel de ceite clientèle.

Nous avons donc élaboré et expérimenté au cours de l'hiver 1989 une intervention de groupe auprès des adolescents auteurs de délits sexuels. Cette expérimentation s'inscrit dans le mandat maintenant reconnu du Service aux

2. LAFORTUNE, Denis (1989), Recueil des textes de la journée d'étude sur l'adolescent auteur d'agression sexuelle, Boscoville, Montréal, mars.

3. BOUFFARD, Michel et al. (1987), Étude exploratoire, Centre de services sociaux de Québec, Québec.

4. DUMAIS, Jacques et al. (1988), Propositions d'orientation..., Centre de services sociaux de Québec, Québec. 
jeunes contrevenants, de prendre en charge les adolescents auteurs de délits sexuels. La nature de ce mandat est déterminée par la déclaration de principes de la Loi sur les jeunes contrevenants : rappelons que cette loi stipule que les adolescents doivent assumer la responsabilité de leurs délits, sans pour autant être assimilés aux adultes. Elle précise également que la société doit pouvoir se protéger contre toute conduite illicite, et enfin que la situation des jeunes contrevenants requiert surveillance, discipline et encadrement et que l'état de dépendance où ils se trouvent, leur degré de développement et de maturité leur créent des besoins spéciaux qui exigent conseils et assistance ${ }^{5}$. L'approche éducative que nous avons développée réfère à la dimension d'aide de notre mandat et se veut complémentaire aux mesures de neutralisation et de contrôle, ainsi qu'aux autres mesures d'aide que peut nécessiter la situation de ces adolescents.

Notre intérêt pour travailler auprès de cette clientèle d'adolescents présentant des désordres sexuels qui généralement provoquent, par la nature même de leur inconduite, des sentiments de gêne, de peur ou même de rejet, s'est développé à travers notre pratique quotidienne, à travers les connaissances que chaque situation référée nous a apportées sur ces jeunes.

Nous ne sommes pas totalement à l'abri des réactions angoissées que peuvent susciter dans la population les délits d'ordre sexuel, mais il nous faut «dépasser» le symptôme que représente le geste d'abus, pour nous centrer plutôt sur le sens de celui-ci. Nous avons réalisé qu'il s'agissait d'adolescents qui n'ont souvent pas rencontré dans leur milieu d'origine les conditions nécessaires au développement harmonieux de leur personnalité, ni à celui de leur capacité d'échange avec l'autre, qu'il soit affectif ou sexuel.

Ces adolescents, qui sont parmi les plus sujets à la désapprobation sociale, nous interpellent dans nos valeurs professionnelles, car c'est avec eux que nous devons maintenant actualiser le principe du droit au traitement. Nous sommes partisans de l'intervention différentielle, de l'intervention qui tient compte des besoins spécifiques de chacun, et cela, dans le respect de notre mandat légal de prévenir toute nouvelle conduite antisociale.

Car les adolescents présentant des désordres au plan de la conduite sexuelle ont des besoins spéciaux, différents de ceux des adolescents auteurs de délits de type acquisitif. La réponse à ces besoins doit correspondre à l'état psychologique dont l'agir sexuel est symptomatique. Car «l'élan sexuel (qu'il soit agi ou fantasmé) s'inscrit dans un rapport à l'autre qui est spécifique et qui renvoie encore au sens qu'a «l'objet» à l'intérieur du fonctionnement

5. Loi sur les jeunes contrevenants, LRC 1985, chapitre Y-1, article 3. 
psychique de tel individu ${ }^{6}$. Ainsi par exemple, et nous référons ici à la typologie de l'abuseur sexuel développée par Van Gijseghem ${ }^{7}$, des activités sexuelles avec de jeunes enfants, que l'on nomme souvent pédophilie, peuvent tout aussi bien être le fait d'un individu carencé, qui recherche l'affection de «l'objet», que le fait d'un individu pervers qui «utilise» alors l'enfant pour réaliser son rêve narcissique.

La réalisation du mandat confié appellera donc des interventions différentes, selon la signification du geste d'abus ou du désordre de la conduite sexuelle. Toute la gamme des services disponibles aux adolescents peut donc être sollicitée. L'intervention de groupe développée s'ajoute à cette gamme. Elle est une des réponses possibles aux besoins de ces adolescents.

Ce que l'on appelle le désordre sexuel est, comme le dit Van Gijseghem ${ }^{8}$, une forme d'exutoire au conflit interne vécu par un individu. Nous croyons qu'il faille répondre à la spécificité de cet exutoire, c'est-à-dire à la sexualisation du conflit, en offrant un programme éducatif composé de volets d'information, d'éducation et de croissance personnelle. Le but visé sera de contribuer à l'adoption d'un comportement sexuel mieux adapté chez ces adolescents en quête d'une identité et possédant peu d'habiletés au plan des relations interpersonnelles.

Nous sommes bien conscients que la délinquance sexuelle à l'adolescence est encore un phénomène méconnu. Toutefois, il s'agit là d'une réalité bien présente dans le quotidien des intervenants travaillant auprès des jeunes contrevenants. Par l'intervention spécifique auprès de cette clientèle, nous souhaitons apporter une contribution, si modeste soit-elle, au développement de la connaissance de cette problématique.

\section{LA PROBLÉMATIQUE}

La délinquance sexuelle se définit par rapport aux normes prévalant dans une société. Ces normes sont supportées par un cadre légal qui établit clairement les comportements jugés répréhensibles, c'est-à-dire qui portent atteinte aux droits d'autrui.

À l'adolescence, le développement psychosexuel de l'individu est marqué par quatre facteurs importants:

— la puberté entraîne un bouleversement de l'image du corps;

6. GIJSEGHEM, Hubert Van (1988), La personnalité de l'abuseur sexuel, Méridien, Psychologie, p. 71.

7. GIJSEGHEM, Hubert Van, Op. cit.

8. GIJSEGHEM, Hubert Van, Op. cit. 
- la vie affective devient plus intense;

- l'action prévaut sur l'expression verbale;

- le jugement moral est à se consolider'.

À cette étape de sa vie, un individu peut présenter une conduite abusive qualifiée d'«accidentelle». Il peut s'agir d'une conduite d'exploration, d'un surplus d'énergie mal canalisée qui n'indique pas nécessairement une déviation. Cependant, ce comportement sexuel non adapté peut également traduire l'intégration par l'adolescent de valeurs familiales et sociales faussées, dans lesquelles la notion d'interdit n'a pas sa place, ou bien la présence chez lui d'un sens moral déficient où les notions du bien et du mal se confondent, ou enfin le cumul de déficits, aux plans personnel, familial et social.

Le sens d'une conduite sexuelle repose sur la perception qu'a l'individu de lui-même et des autres. Un individu qui a développé une perception de soi si négative et une crainte de l'autre si forte qu'il est incapable d'échanges interpersonnels est un individu incapable de trouver une réponse à ses besoins (affectifs et sexuels) dans une rencontre égalitaire avec l'autre. Bill Marshall, de l'Université Queen's de Toronto, a identifié, au cours de ses 16 ans d'intervention auprès des abuseurs sexuels adultes, les besoins qui peuvent supporter la conduite sexuelle abusive:

- recherche de gratification physique;

- réconfort contre des tendances dépressives;

- réduction de l'anxiété (souvent élevée);

- réassurance de l'identité masculine;

- confirmation de l'amour d'un partenaire;

— atténuation de la souffrance d'un manque d'intimité relationnelle ${ }^{10}$.

Il faut donc dégager la signification de la conduite d'abus de l'adolescent pour lui offrir des alternatives valables de satisfaction à ses besoins.

La délinquance sexuelle peut prendre diverses formes. Rappelons ici les différents types de délits sexuels commis par les adolescents référés à nos services:

— Il y a d'abord les agressions sexuelles hétéro ou homosexuelles envers les enfants. Elles se traduisent soit par la manipulation ou la demande de manipulation des organes génitaux, soit par la pénétration vaginale ou anale.

9. LAFORTUNE, Denis (1987), Colloque provincial sur les abus sexuels, TroisRivières, mai.

10. CENTRE DE SERVICES SOCIAUX DE QUÉBEC, Colloque du Service d'aide à l'enfance du Nouveau-Brunswick, Frédéricton, 1988, Résumé des conférences de Bill Marshall par Cyril Touchette, Québec, 1988, pp. 6-7. 
- L'inceste est aussi une forme de délinquance sexuelle commise par ces adolescents. Il s'agit ici des contacts à caractère sexuel entre les membres d'une même famille, principalement entre frère et sœur.

- La déviance sexuelle de nos adolescents s'exprime aussi par le viol, défini comme un contact sexuel non désiré par l'autre, réalisé avec contrainte ou violence.

- L'exhibitionnisme est une autre forme de déviation rencontrée. Il s'agit d'une pratique, masculine et hétérosexuelle, d'exposition volontaire des organes génitaux.

\section{LA CLIENTÈLE}

Deux inventaires de la clientèle des adolescents référés en raison de comportements sexuels déviants ont été réalisés au Centre de services sociaux de Québec. Soixante-deux (62) dossiers ont alors été recensés, soit 22 en $1986^{11}$ et 40 en $1988^{12}$.

Le portrait qui se dégage de cet inventaire est le suivant:

- L'adolescent abuseur est de sexe masculin (97\% des références), d'un âge moyen de 15 ans.

- Les victimes sont majoritairement de sexe féminin et l'écart d'âge moyen entre la victime et l'abuseur varie de 6 à 12 ans.

- Il existe un lien significatif entre l'abuseur et la victime dans $93 \%$ des cas: liens familiaux, relations de voisinage ou encore liens établis dans un travail de gardiennage auprès de jeunes enfants.

- Les abus commis sont surtout des gestes d'attouchement, de masturbation et de fellation, alors que les agressions sexuelles graves (avec violence) constituent une faible partie des références.

- $50 \%$ des abuseurs ont eux-mêmes été victimes d'abus sexuels.

- Au plan social, les aủolescents référés fréquentent l'école, mais ils ont peu ou pas d'amis.

- Au plan psychologique, les adolescents évalués présentent une faible estime et une image négative d'eux-mêmes. Ils vivent de fortes tensions internes, ne trouvent pas de réponses satisfaisantes à leurs besoins. Leur agressivité est faible, on constate plutôt une grande dif-

11. BOUFFARD, Michel et al. (1987), Etude exploratoire, Centre de services sociaux de Québec, Québec.

12. DUMAIS, Jacques et al (1988), Propositions d'orientation..., Centre de services sociaux de Québec, Québec. 
ficulté d'affirmation. Il y a aussi une forte inhibition des sentiments et des pulsions, une crainte des relations interpersonnelles et donc une grande solitude affective.

- L'anxiété est élevée chez ces adolescents, mais elle n'est pas liée aux gestes d'abus commis. Ils ont plutôt une attitude défensive par rapport à leurs inconduites, recourant à la normalisation, à la banalisation.

\section{PHILOSOPHIE D'INTERVENTION}

Notre conception de l'intervention auprès des adolescents présentant des désordres sexuels tient compte du processus développemental particulier à l'adolescence.

Appréhender ainsi le comportement sexuel, qu'il soit ou non approprié, nous permet de penser à l'adolescent en fonction de ses capacités individuelles d'adaptation et aussi de son potentiel de croissance au plan de ses ressources personnelles.

Nous croyons donc qu'un soutien adéquat, associé à une information objective et réaliste, contribue à développer des attitudes plus appropriées chez l'adolescent, réveille chez lui le potentiel d'évolution endormi et favorise ainsi une meilleure adaptation sociale. Considérant le niveau de maturité spécifique à l'adolescence, et encore davantage celui présenté par notre clientèle, sa capacité limitée d'introspection jointe à sa difficulté d'engagement đans un processus à long terme, nous préconisons une approche de type éducatif qui tienne compte de tous ces facteurs.

Les adolescents participant à l'intervention de groupe doivent acquérir des connaissances sexuelles plus justes, développer des perceptions et des attitudes plus adéquates, prendre conscience de leurs besoins affectifs et identifier des moyens appropriés d'y répondre. Ils doivent développer un plus grand sentiment de «compétence sexuelle». Nous croyons que ces apprentissages ne peuvent se faire que sur un mode positif. Ces adolescents ont en effet besoin de se construire une identité positive, eux qui n'osent s'affirmer, qui n'osent se lier aux autres, en raison d'une perception négative, dévalorisée d'eux-mêmes. Il ne faut donc pas que leur participation au groupe soit un nouvel échec, c'est-à-dire une expérience difficile qui vienne leur confirmer cette image négative d'eux-mêmes, mais plutôt qu'il s'agisse d'une expérience enfin réussie et enrichissante. Il nous faut donc être attentifs à leur méfiance, à leur crainte, à leurs limites, et intervenir sur ce plan pour s'assurer qu'ils s'intègrent à la démarche et en profitent le plus possible. L'opposition, quand elle se manifeste, doit être comprise dans sa dimension défensive, la passivité 
doit être reçue comme l'expression de leurs difficultés interpersonnelles, de leur crainte de l'autre.

Le recours à l'intervention de groupe, en complément à l'intervention individuelle, présente selon nous certains avantages reconnus. Le groupe de pairs favorise la socialisation. Ce facteur s'avère important pour nos adolescents qui tendent à s'isoler et à éviter toutes relations interpersonnelles égalitaires. Il permet aussi le support et la confrontation entre eux, de même que la diminution des résistances, d'une façon plus efficace qu'en intervention individuelle. Le groupe fournit également l'occasion d'interactions et d'apprentissages multiples, tant au plan relationnel qu'au plan des connaissances. Encore là, nos adolescents présentent souvent des déficits concernant ces aspects, déficits qui peuvent être avantageusement comblés par le groupe.

\section{OBJECTIFS DE L'INTERVENTION}

L'objectif général de l'intervention vise le développement de comportements sexuels plus responsables et mieux adaptés à la réalité sociale, dans un contexte de soutien et favorable à l'émergence d'une identité plus positive. Les trois aspects touchés par cet objectif sont l'information, l'éducation et la croissance personnelle. Chacun des thèmes sélectionnés, pour les douze ateliers du programme, réfère à l'un ou l'autre de ces aspects.

L'information, qui vise le savoir, la connaissance, est abordée de façon à transmettre une information juste et objective sur :

- la sexualité (physiologie, maladies transmises sexuellement, affectivité);

- les normes sociales en vigueur (aspect légal).

L'éducation fournie vise à corriger les perceptions et les attitudes de ces adolescents en abordant:

- le sens donné au contact sexuel;

- l'impact, chez autrui, de leur comportement sexuel;

— l'anxiété créée par l'établissement de relations interpersonnelles.

La dimension de croissance personnelle vient favoriser:

- la reconnaissance par les adolescents de leurs besoins affectifs et sexuels;

- la prise de conscience de leurs difficultés interpersonnelles;

— l'expression de leur vécu intérieur (désirs, craintes, questionnement). 


\section{CADRE D'INTERVENTION}

\subsection{PRÉALABLE D’ADMISSION AU GROUPE}

L'intervention s'adresse à des adolescents ayant été reconnus coupables de délits à caractère sexuel ou présentant des troubles de comportement associés à une problématique sexuelle et dont la sécurité ou le développement est jugé compromis par le Tribunal de la jeunesse. Par le biais de la Loi sur les jeunes contrevenants et de la Loi sur la protection de la jeunesse, nous pouvons en effet être en contact avec les multiples manifestations des désordres sexuels à l'adolescence.

La participation au programme d'intervention de groupe doit être ordonnée par le Tribunal de la jeunesse. C'est donc dire que nous excluons toute participation sur une base volontaire. Il nous semble en effet illusoire d'attendre d'un adolescent aux prises avec des difficultés au plan de l'expression de sa sexualité, qu'il reconnaisse ses difficultés, qu'il soit motivé à les comprendre et à les régler, alors qu'il présente généralement une réaction très défensive, basée sur la justification ou même la négation. Ce sont aussi, fréquemment, des adolescents peu habiles à s'exprimer, difficilement capables de relations interpersonnelles; ils peuvent donc vite tenter de se soustraire à l'expérience d'un groupe de pairs abordant un sujet comme la sexualité. Cela n'exclut cependant pas la nécessité de favoriser leur adhésion à la démarche, par une animation aftentive aux capacités et aux besoins de chacun.

Le deuxième élément considéré est celui de la capacité intellectuelle suffisante pour participer aux activités d'un groupe d'adolescents. Un trop grand déficit au plan intellectuel ne permet pas les acquisitions visées par notre intervention, tout en «alourdissant» la démarche du groupe.

Le troisième aspect est celui de la capacité de fonctionnement en groupe. Cette capacité est établie selon le degré d'opposition et de méfiance présenté par l'adolescent et selon la qualité de son contact avec la réalité. Par exemple, nous ne pouvons pas, par l'intervention de groupe, vaincre des résistances si fortes qu'elles ne s'expriment que par un mode relationnel agressif ou encore par un mutisme total. Il faut reconnaître que certaines situations nécessitent une intervention plus intensive, que ce soit par le biais des programmes de rééducation des centres d'accueil ou encore le traitement médical.

Compte tenu des étapes du développement à l'adolescence, ainsi que de la réalité de la clientèle déjà référée au Service aux jeunes contrevenants, nous limitons notre intervention aux adolescents âgés de 14 à 18 ans, favorisant ainsi une certaine homogénéité du groupe. Il peut cependant y avoir exception 
à cette règle, particulièrement pour les jeunes de plus de 18 ans qui présentent un retard important de développement.

\subsection{COMPLÉMENTARITÉ DES INTERVENTIONS}

L'intervention de groupe se veut une mesure complémentaire à l'intervention individuelle effectuée dans un cadre légal. Pour que cette complémentarité existe, il est donc nécessaire de s'assurer de la collaboration des intervenants responsables de l'intervention individuelle. Il est en effet important d'échanger nos perceptions de la situation de l'adolescent participant au programme, autant pour adapter la démarche de groupe à cette situation, que pour la compléter par des indications d'intervention individuelle. On peut imaginer par exemple la situation d'un adolescent qui présente une forte attitude d'isolement, de retrait social, en raison de ses difficultés interpersonnelles. Il faut alors que l'apprentissage en groupe soit complété par une intervention individualisée visant l'intégration de l'adolescent à une activité sociale et supportant le maintien de sa participation. L'intervention de groupe vient donc foumir des «moyens» à cette démarche d'intégration, alors que la participation de l'adolescent à une activité sociale lui fournit une occasion d'intégrer les apprentissages faits en groupe.

La complémentarité des deux types d'intervention se retrouve aussi au sein du système familial. Nous intervenons directement auprès des parents pour rechercher leur collaboration à la démarche qu'entreprennent leurs adolescents. Cette collaboration doit se traduire, dans le quotidien familial, par des attitudes parentales adéquates envers les adolescents, plus particulièrement par rapport à la participation à la session de groupe. Les adolescents se sentent alors appuyés dans leurs efforts, ou du moins ne vivent pas d'entrave à leur motivation et à leur implication. L'intervention individuelle, sur ce plan, est donc capitale.

Pour les adolescents en situation de retrait familial, il y a aussi nécessité d'assurer une collaboration formelle avec les intervenants du milieu substitut.

\subsection{THÈMES ET TECHNIQUES}

La première expérimentation de notre programme d'intervention de groupe auprès des adolescents présentant des désordres sexuels s'est déroulée sur une période de 12 semaines. Comme cette démarche poursuivait des objectifs bien spécifiques, il nous apparaissait préférable de limiter ainsi sa durée, afin de nous assurer de la meilleure participation possible des adolescents. En leur présentant un programme de courte durée, nous amoindrissons «le choc» et obtenons plus facilement leur engagement à une dérnarche qui, bien qu'intensive, était bien délimitée. 
Parmi les thèmes retenus, certains traitaient de la physiologie sexuelle, de la contraception et des maladies transmises sexuellement. D'autres, plus nombreux, touchaient les croyances et idées par rapport à la sexualité, les expériences et fantasmes sexuels, les impacts des abus sexuels chez les victimes. Nous avons aussi abordé l'aspect des relations affectives et l'orientation sexuelle, et insisté sur le développement de l'imaginaire. L'information légale fut aussi considérée dans le cadre d'un atelier. Nous avons tenté enfin de faire apprécier la relaxation aux participants. Les autres rencontres ont été réservées au démarrage du groupe et à l'évaluation de la session.

Il va sans dire que l'intervenant responsable du groupe doit être sensibilisé au vécu de ces adolescents; il doit être bien conscient de ses propres valeurs sur la sexualité et il doit se sentir à l'aise pour discuter de problèmes sexuels avec les jeunes impliqués. Ces préalables nous apparaissent essentiels pour dépasser le comportement présenté et comprendre les besoins qu'il sous-tend, pour établir un climat relationnel favorable à la croissance de l'adolescent et pour que l'intervenant puisse servir lui-même de figure de référence au plan relationnel, tout en fournissant au jeune l'occasion de travailler ses difficultés de communication. Les techniques d'animation utilisées dans cet esprit ne constituent donc qu'un moyen, la relation étant la base, pour se centrer sur le vécu des adolescents et solliciter, le plus possible, leur participation.

Dans le cadre de notre première expérimentation, nous avons surtout privilégié des techniques qui favorisaient la communication et l'expression. Le dessin, la lettre anonyme, les échanges en dyade, l'appel à tous, les jeux de rôle, l'audiovisuel ont été utilisés selon les besoins du groupe et selon les thèmes abordés. Nous avons aussi eu recours à la centration pour favoriser une meilleure participation. La reformulation pour synthétiser et clarifier une idée émise, l'information pour réajuster les distorsions cognitives ou combler les méconnaissances, l'universalisation pour dédramatiser une situation ou faire baisser l'anxiété, voilà une liste non exhaustive de techniques qui ont été utilisées dans le cadre des ateliers.

Bien que les techniques «d'expression anonyme» aient été privilégiées au départ, nous avons pu les délaisser progressivement au profit d'une expression plus directe. Le degré de sécurité des participants et le niveau de cohésion du groupe nous ont en effet permis de le faire.

En raison de la gamme des sujets traités tout au long de la session de groupe, nous avons eu recours à des collaborations spéciales pour quelquesuns des ateliers. L'information légale a donc été transmise par un avocat, les deux volets de l'éducation sexuelle ont été assumés par une sexologue et par un médecin, la prise de conscience du corps a été initiée par un psychologue spécialisé en approche corporelle alors que la dimension de la vie affective 
à l'adolescence a été présentée par une sexologue reconnue pour son travail de vulgarisation auprès des adolescents. Le recours à ces experts ne s'est toutefois pas traduit par la présentation de conférences auxquelles assistaient passivement les adolescents. Nous avions préalablement précisé à chacun des collaborateurs le contenu de l'atelier et recherché avec eux les techniques d'animation les plus adaptées. Les deux animateurs ont toujours été présents à chacune des rencontres, pour contribuer à l'animation et assurer la continuité de la démarche.

\section{PREMIÈRE EXPÉRIMENTATION}

\subsection{PROFIL DES PARTICIPANTS}

Pour cette première expérimentation, le groupe se composait de six adolescents âgés de 14 à 20 ans. Tous nous étaient référés par le Tribunal de la jeunesse par le biais d'une condition spécifique contenue dans l'ordonnance émise en vertu de la Loi sur les jeunes contrevenants. Deux de ces adolescents étaient hébergés en centre d'accueil alors que les quatre autres résidaient dans leur famille. Notons que trois d'entre eux avaient intégré le marché du travail, alors que les trois autres fréquentaient l'école. Le groupe comptait des frères jumeaux.

Les gestes commis consistaient en attouchements, masturbation, fellation, pénétration. Dans trois des cas, l'écart d'âge entre la victime et l'abuseur était de plus de 9 ans. Les trois autres abus, de nature homosexuelle, présentaient un écart d'âge abuseur/victime de 8 à 1 an.

Des différentes évaluations réalisées auprès de ces adolescents se dégagent des caractéristiques communes: blocages cognitifs se traduisant par des difficultés d'apprentissage, faiblesse de l'affirmation de soi entrainant une vulnérabilité sociale, méconnaissance sexuelle importante, forte inhibition, faible capacité relationnelle observable, entre autres, par leur isolement social.

\section{2 ÉVOLUTION DU GROUPE}

Nous voulons faire ressortir dans cette partie les éléments les plus significatifs de cette première expérimentation, ceci tant au plan «statique» des rencontres (les thèmes, l'animation) qu'au plan «dynamique» (le cheminement des participants à l'intérieur du cadre proposé). Conscients de l'interdépendance de ces deux dimensions, les commentaires toucheront donc l'ensemble de la démarche. 


\subsubsection{Critique de la démarche}

Lorsque nous considérons les divers thèmes retenus pour les rencontres de groupe, différentes observations se dégagent. D'abord, la plupart des adolescents constituant le groupe n'avaient pas, en dehors des abus commis, de vie sexuelle active. Ainsi, dans le cadre de l'atelier portant sur la contraception et les maladies transmises sexuellement, le message se résumait donc à la nécessité de protection pour soi et pour l'autre, ce qui aurait très bien pu être dit lors de la rencontre sur la physiologie sexuelle. Car au-delà de l'information transmise, s'ajoute un objectif d'éducation: faire prendre conscience aux adolescents que toute relation sexuelle satisfaisante repose sur le respect de soi et de l'autre.

Le malaise de ces adolescents à aborder la sexualité s'est aussi vérifié. Il devient donc important de maintenir les thèmes qui visent à dédramatiser celle-ci. Il leur faut aussi développer leur imaginaire. Il nous faudra aussi davantage nous attarder à leur perception de la femme. Cette première expérimentation nous a confrontés à l'intégration par nos participants de stéréotypes clairement définis, où la fille très sexuée est associée à une «fille facile, vulgaire», alors que l'image de la «bonne fille» est perçue comme asexuée. Pour eux, la sexualité constitue un élément à part plutôt qu'un aspect de la globalité de la personne. Il s'avère donc nécessaire de corriger leurs fausses perceptions pour favoriser une réponse plus adéquate à leurs besoins.

Il va sans dire que certains thèmes, davantage liés à leur inconduite (exemple: les impacts vécus par la victime) augmentent leurs difficultés au plan de l'expression; les résistances se manifestent alors plus ouvertement. Il est toutefois impérieux de maintenir ces thèmes afin de faire prendre conscience à nos participants des implications de leur geste sur l'autre et aussi de l'inadéquation du moyen utilisé pour répondre à leurs besoins. Il faut certes leur rappeler que leur agir abusif est inapproprié socialement, mais aussi intérieurement, puisqu'en définitive, il ne satisfait pas leurs besoins réels d'aimer et d'être aimés. Il faut aussi tenter de les ramener le plus possible à leurs affects, le fait d'en être coupés constituant une autre de leurs limites à dépasser.

En ce qui concerne les techniques d'animation, certains ajustements devront être effectués. Le recours aux personnes-ressources sera grandement restreint et se limitera à deux rencontres (physiologie sexuelle et information légale). Nous avons pu constater en effet que l'insertion de personnes étrangères au groupe en affectait largement la dynamique et le fonctionnement. Nous envisageons de maximiser la relation animateurs-participants pour atteindre l'objectif visé dans le cadre de ces rencontres. 
Les adolescents ont fait ressortir l'importance de rendre accessible la matière transmise par un langage adapté. Le constat que nous faisons est qu'audelà du sujet abordé, il y a tout l'aspect relationnel (besoin d'attention, d'écoute) qui devient primordial et conditionne l'intégration du message. Nous avons également remarqué qu'une attitude directive, mais néanmoins chaleureuse, donnait de meilleurs résultats au plan de leur participation.

La centration, les visualisations, la relaxation demeurent des techniques à utiliser, en les ajustant aux besoins du groupe malgré la résistance qu'elles peuvent, au départ, susciter. Cette insistance est souvent provoquée par une méconnaissance de ces techniques et l'apprivoisement s'effectue progressivement au fur et à mesure que les exercices sont répétés.

\subsubsection{Cheminement des adolescents}

Lorsque l'on considère le cheminement des adolescents tout au cours de la session, on remarque que les trois premières rencontres ont nécessité davantage de directivité et d'encadrement: rappels à l'ordre plus fréquents, difficultés plus marquées dans l'expression des aspects négatifs, besoin plus grand chez les adolescents d'être rassurés et d'obtenir certaines clarifications.

La quatrième rencontre a constitué un point tournant dans la dynamique du groupe. L'aspect relationnel y a pris plus d'importance, les contacts et interactions s'établissant plus aisément entre les jeunes eux-mêmes et entre jeunes et animateurs. Les ateliers suivants ont confirmé cette évolution, le contact se voulant plus intimiste lors des pauses-café et les adolescents nous exprimant plus clairement et ouvertement leurs satisfactions et insatisfactions par rapport à la démarche entreprise.

En fait, nous avons utilisé les situations vécues dans le groupe pour faire cheminer les participants et illustrer des façons de communiquer et d'entrer en relation.

\subsubsection{Résultats observés}

L'atteinte de l'objectif visé avec cette clientèle, soit le développement d'un comportement sexuel mieux adapté socialement, devrait pouvoir considérer d'autres variables: l'observation de la conduite sexuelle des adolescents participants sur une période plus étendue, le développement de leurs capacités interrelationnelles, l'amélioration de l'image de soi. À ce stade-cí, notre évaluation doit se limiter à l'observation des résultats immédiats de l'intervention, compte tenu des moyens limités de mesure dans le cadre de cette première expérience. 
Les résultats que nous livrons ici sont tirés d'un questionnaire que nous avions préalablement élaboré pour mesurer l'acquisition des connaissances et le changement de croyances. Ce questionnaire était rempli par les adolescents à la première rencontre de groupe ainsi qu'à la dernière. Nous compléterons ces informations par le bilan des observations transmises par le délégué à la jeunesse responsable de l'intervention individúelle et conclurons par l'évaluation faite par les animateurs du groupe quant à l'objectif de croissance personnelle poursuivi par notre intervention.

Nous ne prétendons pas que l'intervention de groupe soit la seule redevable des résultats observés. L'être humain étant un être "social», les sources d'influences sont souvent nombreuses et difficiles à départager. C'est là une limite dont nous sommes conscients.

\subsubsection{Au plan de l'information}

Un des objectifs visés par l'intervention de groupe se formulait ainsi: la transmission d'une information juste et objective sur la sexualité et les normes sociales en vigueur. Trois ateliers concernaient plus spécifiquement cet objectif, soit celui consacré à la physiologie sexuelle, un autre traitant de la contraception et de la protection contre les maladies transmises sexuellement, et un demier relatif aux aspects légaux du comportement sexuel.

Voyons d'abord la moyenne des résultats obtenus par le groupe aux questions rattachées à cet objectif et ensuite, selon les trois thèmes abordés.

\begin{tabular}{lcc}
\hline ATELIER & $\begin{array}{c}\text { Iere PASSATION } \\
(21 \text { février 89) }\end{array}$ & $\begin{array}{c}\text { 2e PASSATION } \\
(3 \text { mai 89) }\end{array}$ \\
\hline Moyenne globale & $73 \%$ & $86 \%$ \\
\hline $\begin{array}{l}\text { Physiologie sexuelle } \\
\text { Contraception et protection } \\
\text { vis-à-vis les MTS }\end{array}$ & $66 \%$ & $86 \%$ \\
Aspects légaux & $66 \%$ & $80 \%$ \\
\hline
\end{tabular}

À la stricte observation des résultats, on pourrait conclure que les adolescents possédaient déjà une bonne connaissance au plan légal et que le changement réalisé ne correspond qu'à $4 \%$. Pour les deux autres thèmes abordés, on note une amélioration de l'ordre de $14 \%$ et de $20 \%$.

Au plan de la physiologie sexuelle, l'amélioration constatée est de l'ordre de $20 \%$. Le questionnaire ne nous permet cependant pas de mesurer la prise 
de conscience de leurs besoins sexuels ainsi que la reconnaissance des besoins de l'autre que visait la transmission de ce type d'information. Nous y reviendrons plutôt au niveau de l'objectif de croissance personnelle. Nous ne pouvons donc ici qu'affirmer que les adolescents ont développé une meilleure connaissance de l'aspect physiologique de la sexualité.

L'atelier sur la contraception et la protection vis-à-vis les maladies transmises sexuellement avait pour principal objectif la prise de conscience du besoin de protection dans les activités sexuelles à risque. Le questionnaire permet de mesurer cette reconnaissance des risques possibles et la connaissance des moyens de protection. L'amélioration des connaissances constatée ne nous permet cependant pas de conclure que les adolescents se protégeront adéquatement contre les risques d'une grossesse non désirée pour leur partenaire et contre les risques de maladies. En effet, on constate, dans le cadre de la problématique du SIDA, que les différentes campagnes d'information menées auprès des clientèles à risque n'ont pas suscité des modifications durables du comportement sexuel et que la "pensée magique» est une protection encore largement utilisée par les adolescents.

Quant aux aspects légaux, les réponses des adolescents témoignent de leur réaction face au dévoilement de leur inconduite sociale et à la réprobation subie. Ils ont, en effet, développé une perception négative de la sexualité et ont exprimé la conviction qu'aucune activité sexuelle ne leur était autorisée. Comme le questionnaire visait à mesurer l'intégration des interdits sociaux, les adolescents ont exprimé lors de la première passation, cette perception que toute vie sexuelle leur est interdite. Si l'on tient compte de l'intérêt démontré pour cette question, si l'on tient compte également de la prise de conscience de leurs besoins sexuels et des éléments de l'intervention visant à supporter la recherche d'une réponse adaptée socialement à ces besoins, nous émettons l'hypothèse que les résultats de la deuxième passation traduisent davantage une connaissance juste et objective qu'une croyance que rien ne leur est permis.

\subsubsection{Au plan de l'éducation}

Par éducation, nous entendons la correction des perceptions et des attitudes des adolescents face à la sexualité et aux relatiotns interpersonnelles. Les ateliers visant cet objectif portaient sur les relations affectives à l'adolescence et l'orientation sexuelle, sur les croyances sexuelles ainsi que sur l'impact chez autrui du comportement sexuel.

Les résultalts moyens obtenus par le groupe sont les suivants: 


\begin{tabular}{lcc}
\hline ATELIER & $\begin{array}{c}\text { 1ere PASSATION }^{\text {(21 février 89) }} \\
\text { Moyenne globale }\end{array}$ & $\begin{array}{c}2^{\mathrm{e}} \text { PASSATION } \\
(3 \mathrm{maj} 89)\end{array}$ \\
\hline $\begin{array}{l}\text { Relations affectives et orientation } \\
\text { sexuelle }\end{array}$ & $83 \%$ & $83 \%$ \\
$\begin{array}{l}\text { Impact du comportement sexuel chez } \\
\text { autrui } \\
\text { Croyances par rapport à la sexualité }\end{array}$ & $75 \%$ & $91 \%$ \\
\hline
\end{tabular}

Les résuitats se sont donc améliorés globalement de près de $20 \%$. C'est au plan des croyances que l'éducation a eu le plus d'impact, la correction étant de l'ordre de $23 \%$ au niveau des réponses portant sur cet aspect.

Nous pouvons dégager de ces résultats qu'une correction importante s'est réalisée au plan des croyances que ces adolescents avaient développées par rapport à la sexualité. Les erreurs de pensée étaient évidentes chez eux. La majorité percevait la sexualité comme quelque chose de néfaste pour eux, niant leurs besoins et ne s'autorisant aucune forme de réponse satisfaisante. À partir des réponses au questionnaire, nous pouvons croire qu'il s'est opéré une normalisation de leurs perceptions face à la sexualité.

L'impact des abus chez les victimes est plus reconnu, mieux identifié alors que l'orientation homosexuelle est mieux comprise. L'intervention de groupe a permis à la majorité des adolescents qui y ont participé de modifier leur compréhension de la sexualité, de leurs besoins sexuels et de développer une prise de conscience des conséquences chez l'autre de leur propre conduite. Sur ce plan, notre expérience nous avait permis de constater leur attitude de négation, ou encore, de minimisation face au traumatisme causé aux victimes. L'objectif visé de comportements sexuels mieux adaptés chez ces adolescents repose d'abord sur cette prise de conscience de leurs besoins et de ceux des autres.

\subsubsection{Au plan de la croissance personnelle}

L'objectif de croissance personnelle visait la reconnaissance par les adolescents de leurs besoins affectifs et sexuels, la prise de conscience de leurs difficultés interpersonnelles et l'obtention de réponses plus satisfaisantes à leurs besoins.

Cet aspect a été évalué à partir de l'appréciation faite par les animateurs et par le délégué à la jeunesse responsable du suivi en probation. 
Appréciation par les animateurs de l'évolution des adolescents dans le groupe:

Nous constatons d'abord que l'intérêt et la confiance se sont progressivement développés pour la plupart des adolescents. Bien que ce soit à des degrés différents pour chacun, leur cheminement a été nettement observable: développement d'une plus grande aisance, capacité d'expression accrue (nombre et qualité d'interventions), amélioration de la qualité des contacts par un plus grand engagement personnel et une personnalisation plus évidente, tant dans les relations avec les animateurs, où l'humour avait sa place, qu'avec les autres participants. La capacité d'affirmation, pour l'un, s'est suffisamment développée pour qu'il soit capable d'exprimer très convenablement à une personne-ressource qu'il souhaitait terminer ce qu'il avait à dire. Un autre tenait à présenter au groupe son amie. L'attitude de certains adolescents, tant par leur implication dans les tâches que par les échanges développés en groupe, a largement contribué au cheminement du groupe.

Le cheminement d'un autre adolescent s'est traduit par le développement d'un plus grand intérêt, intérêt perceptible par une attention plus soutenue. Bien que la gêne soit demeurée très présente (questions en aparté aux animateurs), nous avons quand même pu percevoir une amélioration de sa capacité d'expression dans le groupe. Ainsi, il se risquait parfois à intervenir pour poser des questions ou demander la signification d'un terme. Toutefois, son implication en présence des personnes-ressources se limitait à l'écoute. Sa capacité relationnelle était également freinée par son bégaiement.

Un autre participant avait développé son intérêt et sa confiance lors des premières rencontres, mais son implication a vite plafonné. Sa difficulté d'expression, sa très grande méfiance à l'égard d'autrui, sa peur viscérale de tout et de tous, de même que sa faible capacité intellectuelle, constituaient des barrières importantes à l'intervention de groupe. Sa participation dans le groupe était grandement diminuée par ses résistances et sa faible conscience de ses propres besoins et de ceux des autres.

Chez un autre adolescent présentant, lui, un bon potentiel, nous avons observé un intérêt et une attitude positive constants. Il s'efforçait de répondre aux demandes, très désireux de faire «comme il faut». Sa participation était en fait empreinte de conformisme et de désir de plaire. Ses capacités, nettement supérieures à celles des autres, ne se sont cependant pas actualisées autant que nous l'aurions souhaité, même s'il a assumé un certain leadership au sein du groupe. Il lui faudrait dépasser sa gêne pour mieux satisfaire ses besoins. Son insécurité est encore très forte, ce qui l'amène à rechercher son bien-être en se conformant d'abord aux attentes des autres. 
Appréciation par le délégué à la jeunesse de l'évolution de l'adolescent:

Pour trois des adolescents, les informations transmises par le délégué assumant l'intervention individuelle confirment les observations faites par les animateurs. Dans un cas, on souligne l'énorme difficulté des parents à cheminer positivement. En effet, il s'agit d'une famille qui évite d'aborder certains sujets (relations conjugales, relations dans la fratrie). Seul l'adolescent exprime, et bien souvent à mots couverts, son malaise à composer avec cette réalité. Ce jeune a fait un pas important dans le développement de sa capacité d'affirmation en mettant à distance sa famille. Les commentaires qu'il a transmis au délégué quant à la session de groupe se sont révélés très positifs.

Dans un autre cas, il est confirmé que les limites intellectuelles et personnelles constituaient une entrave sérieuse aux acquis possibles. L'interdit a été transmis, les moyens possibles d'accéder à d'autres types de relation sont connus mais la probabilité d'actualiser ces découvertes demeurent très minces. L'objectif visé se limitait d'ailleurs, outre l'interdit, à lui fournir une information de base sur la sexualité.

Avec un troisième adolescent, le délégué rapporte des changements notables dans la relation établie avec lui : plus grande ouverture, attitude plus cordiale, expression plus claire et plus directe des besoins, et fonctionnement social plus satisfaisant (moins d'isolement, activités plus nombreuses).

Chez un autre participant, le délégué a observé divers changements : il parle plus aisément de sexualité, de ses craintes et de ses projets. Le psychiatre responsable de la thérapie individuelle note des transformations qui se répercutent dans leurs contacts : plus grande aisance, humour fréquent, meilleure affirmation, tant dans son attitude physique que dans son discours. La thérapie s'est terminée en juin, le psychiatre considérant les acquis de l'adolescent suffisants pour s'assumer de façon autonome. On constate aussi qu'il apporte une plus grande attention à son habillement et à son apparence en général et qu'il tend à s'affirmer davantage dans les événements de son quotidien.

Le délégué responsable des jumeaux rapporte d'abord que, chez l'un deux, aucun changement ne s'est manifesté. Son discours se calque sur celui du délégué. Il demeure méfiant, conformiste, utilisant le mensonge et la fuite dans ses communications interpersonnelles.

Pour l'autre jumeau, un cheminement s'est amorcé, malgré le peu de soutien du milieu familial. Concernant ses problèmes de comportement à l'école, il a lui-même demandé une feuille de route et s'y conforme. Il a aussi lui-même entrepris des démarches de traitement pour remédier à son bégaiement et à son énurésie. Il veut qu'on "arrête de rire de lui» et qu'il ne soit pas toujours «obligé de cogner» pour avoir la paix. Ses acquis demeurent 
fragiles et nécessitent appui et stimulation de la part du délégué, mais le niveau de conscience de ses besoins s'est développé, tout comme la possibilité d'apporter de nouvelles solutions aux problèmes rencontrés.

\section{CONCLUSION}

Cette première expérimentation, au Centre de services sociaux de Québec, de l'approche éducative auprès des adolescents auteurs de délits à caractère sexuel, a été très riche en enseignements.

Ainsi, contrairement à nos appréhensions, les adolescents ont démontré un très grand intérêt à la démarche proposée. Leur participation a été active et leur engagement constant. Nous avons aussi constaté qu'un climat de confiance s'est rapidement installé dans le groupe. Cela a facilité les échanges entre les participants et permis le développement d'une autodiscipline à l'intérieur du groupe.

Un autre constat important réalisé lors de cette expérimentation est la réceptivité démontrée par les adolescents aux messages transmis. Nous attribuons leur réceptivité au climat de confiance qui s'est créé dans le groupe, mais aussi au fait que cela répondait à leurs besoins. La reconnaissance de leur droit à la sexualité, la démystification de cette dimension de leur vie et l'enseignement de moyens adaptés pour répondre à ces besoins leur ont permis de laisser tomber leurs défenses et ont soutenu cet intérêt manifesté à chaque semaine.

Bien sûr, avec des adolescents, nous pouvions miser davantage qu'avec des adultes, sur une attitude conformiste et des capacités d'adaptation plus grandes. Toutefois, il est reconnu que les adolescents abuseurs présentent des difficultés relationnelles importantes liées à leur insécurité personnelle. Ils sont souvent en situation de retrait social, ou encore ils n'établissent que des relations superficielles avec autrui. En abordant avec eux la sexualité de façon positive, en nous donnant un mandat d'éducation, nous avons offert à ces adolescents l'occasion de vivre une expérience sociale positive. Ils ont été l'objet d'attention et d'écoute. Ils ont obtenu des réponses satisfaisantes à leurs questionnements, à leurs préoccupations. Ils ont été alimentés par l'intervention réalisée.

Nous pouvons également noter que le regroupement d'adolescents ayant commis des abus sexuels leur a permis de dépasser leur attitude défensive face à leur conduite et face à la sexualité. Cela a facilité la reconnaissance et l'expression de leurs besoins. Il y a eu également des échanges fructueux entre eux, des membres du groupe se chargeant de corriger les erreurs de pensée exprimées par d'autres participants. Cette expérience de groupe a aussi été l'occasion pour eux de se reconnaître une meilleure capaciié relationnelle. 
L'examen des résultats immédiatement observables nous permet de constater que les objectifs poursuivis ont été bien servis par le mode d'intervention utilisé. L'objectif premier de notre intervention, soit celui de l'adoption d'un comportement sexuel mieux adapté, a été abordé en fournissant aux adolescents «l'équipement de base» nécessaire. Au terme des douze rencontres, nous constatons que les participants sont mieux outillés aux plans de la connaissance, de la prise de conscience de leurs besoins et de ceux des autres, ainsi qu'aux plans de l'affirmation de soi et des habiletés relationnelles. Et surtout, «l'interdit» leur a été clairement signifié.

Nous concevons notre intervention comme complémentaire aux autres interventions. Certains adolescents ont d'abord besoin d'être confrontés à leur agir par l'imposition de mesures de conséquences à l'acte. Plusieurs ont aussi besoin d'être aidés individuellement dans une meilleure insertion sociale. On remarque aussi fréquemment la nécessité pour les parents de corriger leurs attitudes, plus particulièrement par rapport à la sexualité, ainsi que celle de modifier le mode de relation développé avec leurs adolescents.

Nous demeurons convaincus que c'est au moyen d'une intervention adaptée à la situation de chacun que la récidive peut être contrée. L'intervention de groupe au plan du développement psychosexuel, souvent nécessaire, ne constitue qu'un élément de la prise en charge des adolescents abuseurs et il est essentiel qu'elle se développe en fonction des besoins réels de la clientèle. C'est pourquoi, tout au long de la session, nous avons questionné tant la forme que le contenu de notre intervention pour l'améliorer et la rendre plus efficace.

\section{RÉFÉRENCES}

AWAD, C. G., E. SAUNDERS et A. LEVENE, «A Clinical Study of Male Adolescent Sexual Offenders", International Journal of Offenders Therapy and Comparative Criminology, pp. 105-115.

BELTRAMI, E., N. COUTURE et C. BOUDREAU (1984), Traitement intensif de la délinquance sexuelle non violente, Sexualité et difficultés d'adaptation, éd. Iris, Montréal.

BOUFFARD, Michel et al. (1986), Étude exploratoire sur les comportements sexuels abusifs chez les adolescents, Centre de services sociaux de Québec, Québec.

CLOUTIER, Richard (1982), Psychologie de l'adolescence, Gaétan Morin, éd., Chicoutimi.

CLAES, Michel (1983), L'expérience adolescente, Pierre Mardage, éd., Bruxelles.

DUMAIS, Jacques et al. (1988), Proposition d'orientation pour l'évaluation et la prise en charge des adolescents abuseurs sexuels, Centre de services sociaux de Québec, Québec.

FINN, L. L. (1983), «What's Normal Adolescent»? dans Clinical Pediatrics, $\mathrm{n}^{\circ} 2$. 
GESELL, Amold et L. FRANCES (1985), L'adolescent de 10 à 16 ans, P.U.F., Psychologie d'aujourd'hui.

GIJSEGHEM, Hubert Van (1978), Éducation sexuelle des personnes en difficulté d' adaptation, Les publications C.Q.E.E.

GIJSEGHEM, Hubert Van (1988), La personnalité de l'abuseur sexuel, Éd. du Méridien, Montréal.

GOUVERNEMENT DU CANADA, Loi sur les jeunes contrevenants, chapitre 110, Ottawa, 1983.

KRYMKO-BRETON, Irène (1984), Le développement affectif normal de l' enfant et de l' adolescent, Gaétan Morin, éd., Chicoutimi.

LAFOREST, Suzette et Richard PARADIS (1989), Approche éducative auprès d'adolescents présentant des désordres sexuels, Centre de services sociaux de Québec. Québec, février.

LAFOREST, Suzette et Richard PARADIS (1989), Bilan de la première expérimentation d'une intervention de groupe selon l'approche éducative, Centre de services sociaux de Québec, Québec, octobre.

LAFORTUNE, Denis (1989), «Joumée d'étude sur I'adolescent auteur d'agression sexuelle», Recueil des textes de présentation, Boscoville, Montréal, mars.

LAFORTUNE, Denis (1987), Résumé d'ateliers portant sur les comportements sexuels abusifs chez les adolescents, Colloque, Trois-Rivières, 1987. Par Suzette Laforest, Québec.

LEMAY, Michel (1973), Psychopathologie juvénile, tome 2, coll. Pédagogie psychosociale, éd. Fleunus, Paris.

MANSEAU, Hélène (1983), Délinquants sexuels, évaluation et traitement, Université du Québec à Montréal, novembre.

MARSHALL, B. (1988), Le traitement des abus sexuels envers les enfants : par où commencer, Résumé d'une conférence par Cyril Touchette, Québec, juin.

O'BRIEN, Michaël et Bera WALTER (1986), «Adolescent Sexual Offenders: A Descriptive Typology» in Preventing Sexual Abuse, vol. 1, n 3, automne. 